\title{
Offenbar besteht ein enger Zusammenhang
}

Fragestellung: In welchem Zusammenhang stehen spezifische Schlafprobleme und selbstverletzendes Verhalten im Jugendalter?

Hintergrund: Risikofaktoren für selbstverletzendes Verhalten beinhalten Umweltfaktoren und psychologische Faktoren. Selbstregulatorische Prozesse und insbesondere die Schlafregulation scheinen eine bedeutende Rolle im Rahmen von selbstverletzendem Verhalten zu spielen. Schlafprobleme erwiesen sich dabei als von psychischen Erkrankungen unabhängige Prädiktoren für Selbstverletzung und Suizid. Ob spezifische Ausprägungen von Schlafproblemen das Auftreten von selbstverletzendem Verhalten beeinflussen, ist bislang unklar.

Patienten und Methodik: Im Rahmen einer norwegischen, populationsbasierten Studie zur seelischen Gesundheit wurden 10.220 Jugendliche zwischen 16 und 19 Jahren zu selbstverletzendem Verhalten und Schlafproblemen befragt. Dabei wurde zwischen Insomnie im Sinne von Schwierigkeiten beim

Hysing $M$, Sivertsen B, Stormark KMet al. Sleep problems and self-harm in adolescence. Br J Psychiatry 2015; 207: $306-12$ Ein- oder Durchschlafen, im Bett verbrachter Zeit, Einschlafzeit sowie Erwachen nach Einsetzen des Schlafs unterschieden. Zudem wur- den demografische Daten, Symptome einer Depression, Perfektionismus und Symptome einer ADHS erhoben.

Ergebnisse: Die Teilnehmerquote lag bei 53\%, $98 \%$ der Befragten waren Schüler. 7,2 \% berichteten von selbstverletzendem Verhalten, wovon $55 \%$ bereits zwei oder mehr Episoden erlebt hatten. Ein signifikanter Zusammenhang zwischen Schlafdauer und Selbstverletzung konnte gezeigt werden: So war die durchschnittliche Schlafdauer um etwa eine Stunde kürzer in der Gruppe der Jugendlichen mit selbstverletzendem Verhalten (5,33 vs. 6,29 Stunden; $\mathrm{p}<0,001)$. Signifikante Unterschiede zeigten sich auch bei Einschlafzeit, Erwachen nach Einsetzen des Schlafes und Schlafmangel. Ferner wurde eine Dosis-Wirkungs-Beziehung zwischen dem Ausmaß der Schlafprobleme festgestellt: So litten $48 \%$ der Jugendlichen mit mehrmaligen selbstverletzenden Episoden an Insomnie, während dies auf $37 \%$ derjenigen mit nur einer Episode zutraf ( $\mathrm{p}<0,001)$. Depression erklärte einen Teil des Zusammenhangs zwischen Schlafproblemen und Selbstverletzung, aber nicht Perfektionismus und ADHS.

Schlussfolgerungen: Die Studie zeigt einen starken Zusammenhang zwischen Schlafproblemen und selbstverletzendem Verhalten. Interventionen für betroffene Jugendliche sollten Schlafprobleme als Therapieziel mit einschließen.

\section{- Kommentar von Jessica Mattivi und Klaus Lieb, Mainz}

\section{Weiterer Forschungsbedarf zum Praxistransfer notwendig}

Die populationsbasierte Querschnittstudie (Evidenzniveau III-IV) konnte den bereits zuvor in der Literatur beschriebenen Zusammenhang zwischen Schlafproblemen und selbstverletzendem Verhalten bei Jugendlichen bestätigen und aufzeigen, dass dieser Zusammenhang trotz Berücksichtigung von Depression und weiterer Covariablen signifikant bleibt. Es handelt sich hier jedoch um die erste Studie, die in diesem Zusammenhang eine Vielzahl von Parametern bezüglich des Schlafes untersuchte und somit eine differenzierte Betrachtung ermöglicht. Es ist jedoch zu beachten, dass die erhobenen Parameter allesamt mittels Fragebogen erhoben wurden und somit auf Selbsteinschätzung der Befragten beruhen, was ein gewisses Risiko für systematische Fehler bedingt. Zudem können aufgrund des Studiendesigns keine Aussagen zu Kausalität oder Zeitverlauf getroffen werden. Folgerichtig betonen die Autoren die Notwendigkeit der Durchführung von Längsschnittstudien. Die in der vorliegenden Studie geschätzte Prävalenz von selbstverletzendem Verhalten liegt mit 7,2\% unterhalb dessen, was vorhergehende Arbeiten festgestellt hatten [1]. Aufgrund des Umstands, dass Nichtteilnehmer oftmals eine ausgeprägtere psychische Problematik aufweisen [2], sollte bei der Frage nach der Generalisierbarkeit der Ergebnisse die Teilnehmerquote von $53 \%$ bedacht und die Möglichkeit der Unterschätzung des Vorkommens von selbstverletzendem Verhalten, Schlafproblemen und depressiver Symptomatik berücksichtigt werden, wie auch die Autoren einräumen. Nichtsdestotrotz bietet die vorliegende Studie wertvolle Anregungen zur Hypothesengenerierung für zukünftige Forschung auf dem Gebiet. Die Berücksichtigung von Schlafproblemen innerhalb von Interventionen bei selbstverletzendem Verhalten erscheint im Rahmen von entsprechenden klinischen Studien sinnvoll.
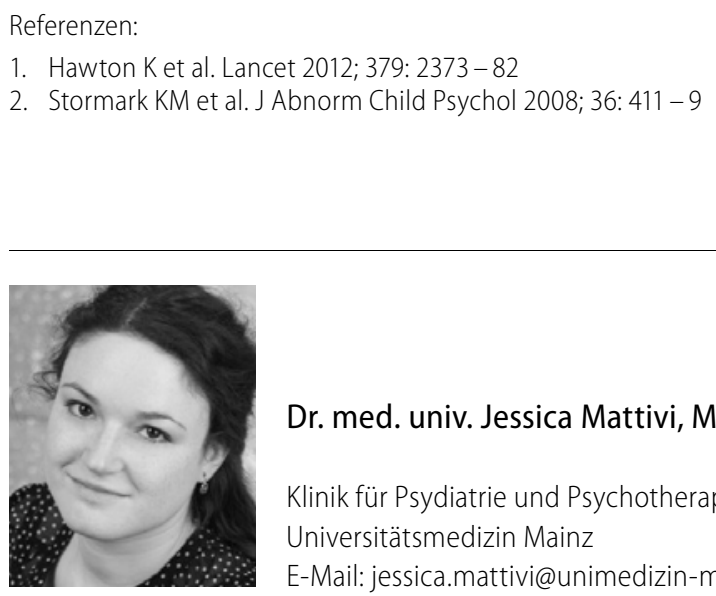

Dr. med. univ. Jessica Mattivi, Mainz

Klinik für Psydiatrie und Psychotherapie, Universitätsmedizin Mainz

E-Mail: jessica.mattivi@unimedizin-mainz.de 